\title{
The Geometry of Air: Large-Scale Multi-colour Robotic Additive Fabrication for Air-Distribution Systems
}

\begin{abstract}
This research investigates the integrated design, robotic fabrication and materialisation of novel air-distribution systems for contemporary office environments. Specifically, the research focuses on the integration of computational design, multiobjective optimisation and the development of novel approaches and techniques for large-scale 3D printed module systems for low embodied-energy air-ducts in the context of a pilot study for an open office environment. By investigating the interrelationship of polymer extrusion, material behaviour, computational design, computational fluid dynamic (CFD) simulation and multi-objective optimisation, the research leverages the capacities of each of these methods in order to create highlydetailed geometries that are optimised for air distribution in order to create locally differentiated thermal comfort. The paper contributes to and further extends existing research in the domain of large-scale $3 \mathrm{D}$ printing by presenting a series of developed novel approaches and techniques combining the diverse methods above to address the plural performance demands of low embodied-energy air distribution systems. It presents empirical research towards air simulation, geometry optimisation and bespoke fabrication for a high-quality, customised and resilient air-distribution system to support the flexibility of contemporary office environments and reduce the adverse per capita environmental impact of cities through increased resource-use efficiency and substantial waste and embodied energy reduction.
\end{abstract}

\section{Introduction}

The vast majority of office environments are retrofits in existing building stock. Within conventional A-grade multi-storey office buildings, services contribute on average $33 \%$ to the embodied carbon footprint of the overall building, with air-conditioning alone constituting $25 \%$. From this portion of the material footprint, ductwork represents the majority at $60 \%$ (Noller 2020). These figures are additionally significant in the context of new workplace organisational models which require flexibility and rapid change within existing building stock. The challenge is to create service systems which are able to support this flexibility and rapid reconfiguration. Moreover, current ducting systems are ill-suited to contemporary office and work environments since tenancy agreements are increasingly short-term leases, which result in frequent changes of the fit out to cater for the requirements of the new tenants. Existing ductwork that cannot be adapted to changing layout requirements is often demolished contributing to an increase in construction waste. Simultaneously, there 
is a clear trend towards excluding suspended ceilings in new office fit outs in order to gain embodied energy savings and increased room height. The resulting exposure of the ductwork provides the potential for visual and individualised thermal benefit to the occupants, though this potential remains currently unrealised. Further, the unsightly nature of existing ductwork systems is significantly dampening the move towards ceiling free offices.

Consequently, this research aims to recalibrate the environmental impact of the built environment, and to drive meaningful action towards decarbonisation and sustainable digital fabrication by proposing an alternative to conventional air-distribution systems. This research focuses specifically on the design, simulation, material and robotic fabrication of a novel air-distribution system. Through a detailed exploration of the affordances of large-scale 3D printing, the research aims to enable the design and fabrication of an air distribution system that is efficient, differentiated to on-site affordances and customisable.

\subsection{Existing material systems and fabrication approaches}

Ductwork and air-distribution systems are most commonly made from thin sheet metal, such as G2 carbon or galvanised steel coils with a typical gauge between 0.6 to $2.0 \mathrm{~mm}$ and are folded into linear rectangular profiles and standard bifurcating elements. Industry processes closely integrate dimensioning, fabrication and automation of linear components. The maximum width of stock material (coil), has resulted in the adoption of a standardised $1400 \mathrm{~mm}$ length for straight sections. Curved, tapered, or bespoke components remain time and labour intensive due to general fabrication constraints and the accompanying demand for manual processing (see Figure 1). The relative cost of non-standard rigid ducts pushes designers to minimise their quantity by distorting overall configurations to favour standard straight elements and by using flexible or semi-flexible ductwork in their place. Both approaches decrease the efficiency of air movement.

Moreover, industrial folding processes - even for simple rectangular cross-sections - frequently feature joint components that leak air, adding to the inefficiency of the resulting thermal system. The implementation of non-differentiated, symmetrical cross-sections - due 
to fabrication constraints - results in highly inefficient airflow across the system. Other limitations extend to a potential fabrication response to variable cross-sections of elements; optimisation of duct geometries for airflow, material distribution or structural requirements; or element specification for connecting parts.
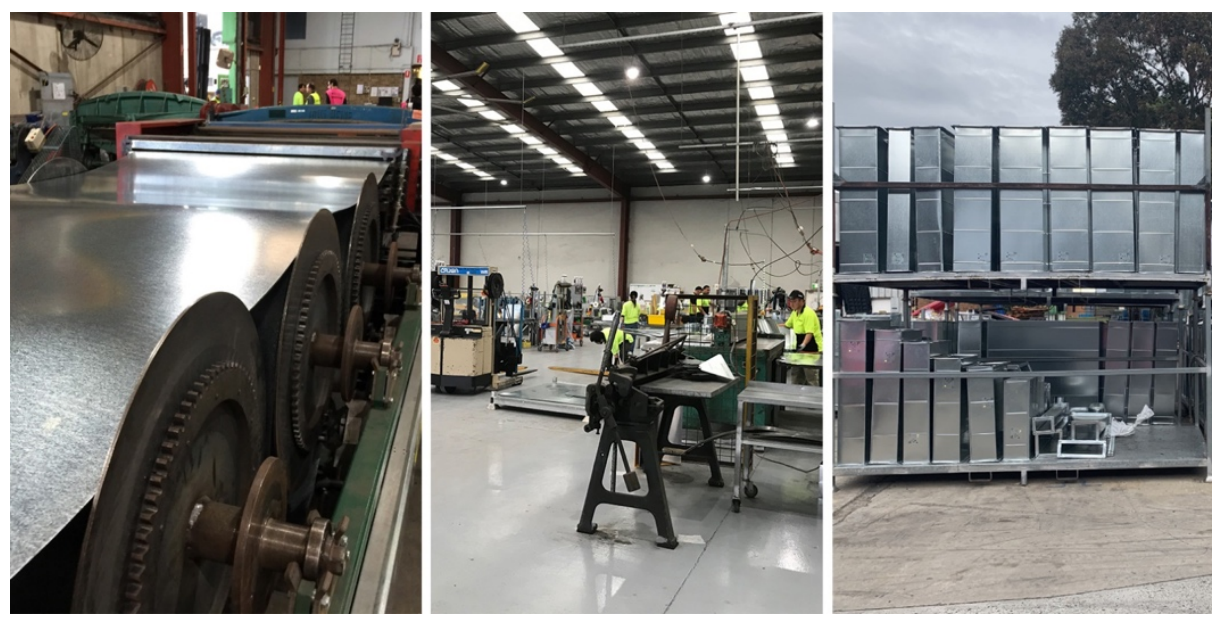

Fig. 1. Current fabrication of steel ductwork showing automated folding of steel coil (left), manual assembly of curved and tapered components (centre) and completed straight components ready for transport to site (right).

In air-distribution systems, solutions vary between integrated and exposed ceiling systems. Negative effects can stem from integrated or suspended ceilings, predominantly in the reduction of floor-to-ceiling height and in the economic and environmental costs of providing the ceiling itself. Metal is a poor thermal insulator and the temperature differential between the un-serviced air space above the ceiling and the air within the ductwork can lead to condensation and energy loss via conduction. As a result, metal ductwork within cavities requires an insulating wrapping, called "lagging". In exposed ceiling air distribution scenarios, there are limited alternative solutions to rigid metal ductwork. Common alternatives include flexi-duct systems (high vulnerability and cost-intensive to install), and fabric ducts (limited structural performance). Whereas the reduction of cost, and environmental impacts, simplification of installation, and thermal performance play a significant role, none of these systems have yet led to the replacement of sheet-metal ductwork or significant reduction of 
suspended ceiling systems, nor do these systems deliver adequate aesthetic benefits (see Figure 2).
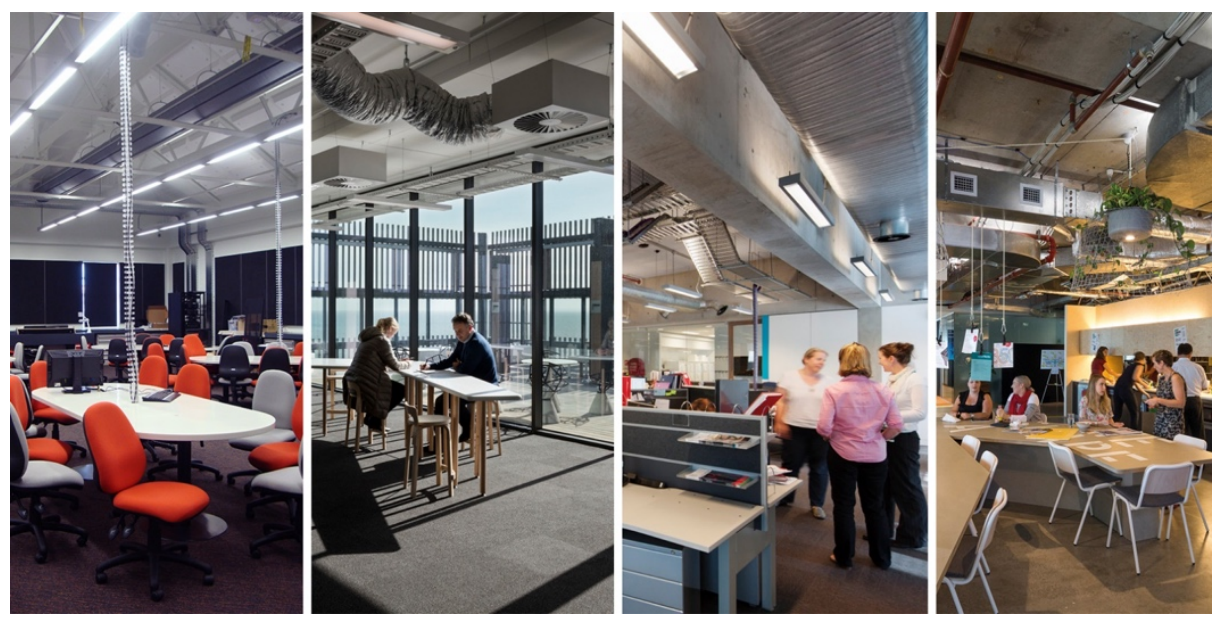

Fig. 2. Existing ductwork systems (left to right). Flexi, rigid, spiral-wound and fabric ductwork.

\section{The Geometry of Air}

\subsection{From low resolution to high resolution geometries}

Standard geometries of existing ductwork and air-distribution systems have to date been optimised for and are limited by the fabrication process and material systems employed. This results in a catalogue of primitive low resolution geometries that are not ideal for airflow, have inefficient air distribution, create environments with low thermal comfort and are of poor aesthetic quality. Harnessing the affordances of computational design and large-scale robotic $3 \mathrm{D}$ printing, the primary research objective of this project is to design a novel air-distribution system that significantly reduces the embodied carbon by investigating the entire life cycle and possible material palette of air-distribution systems. In order to achieve this we explored and developed novel approaches and techniques in large-scale polymer 3D printing that enable the fabrication of: a) aerodynamic and asymmetric crosssections with a continuous tapering geometry that maintains air pressure and velocity; b) geometries for integrated and locally differentiated perforations that respond to inside constrained zones and outside localised thermal environment c) a connection strategy for smooth transitions between duct profiles that change in dimensioning 
relative to airflow; and d) the integration of colour and gradients. These approaches and techniques were applied and tested on-site in a 1:1 scale pilot study in collaboration with BLINDED.

\subsection{Existing approaches to robotic 3D printing}

Research into robotic additive manufacturing methods and specifically 3D extrusion, holds a significant potential to inform design-tofabrication workflows and functionalities of geometries and material systems for the advancement of architecture, design, and construction industries. While there has been significant research into the potential of AM for architecture, to date no research has investigated the application of AM to air-distribution systems. Additive manufacturing has recently moved beyond in-plane mono-material, 2D layer-based methods, towards freeform and 3D differentiated material depositions (Hack et al. 2014; Laarman et al. 2014; Soler et al. 2017). Large scale robotic 3D printed elements for architectural componentry has been extensively investigated across different materials, including coloured PLA (Helm et al. 2015), ABS plastics (Branch Technologies), and concrete (Contour-Crafting, XtreeE), metal (MX3D) or adobe clay printing (IAAC Barcelona). Here, an expansion of form and element geometries, and the optimisation of material systems play a significant role, particularly in response to environmental conditions, and element and texture variations according to local performance requirements. The potential for complex bifurcated geometries has further been explored in medical applications, such as the 3D printing of bifurcated stents (Kim and Lee 2018) or heart replacement (Pataky et al. 2012), and in the aerospace industry for air ducts and fittings in commercial airplanes (Singamneni et al. 2019, Bhate 2015, Lyons 2014).

Beyond this and of interest to this research are particular approaches to multi-material and recycling-oriented additive manufacturing strategies. PET-G emerged as the preferred material as it is safe to work with, has better thermal properties compared to steel, a low shrinkage rate, can meet fire regulations and is an ocean bound plastic that could be reclaimed from existing recycling streams. Recent investigations with this material range from small-scale printing of recycled plastics (Dirk Vander Kooij), gradient colour transitions (Nagami), and large scale PETG printing (Snooks 2019). Furthermore, recyclable PET and PETG are increasingly used in consumer grade 3D 
Printing, with the material being sourced from recycled materials (3DFuel 2020).

\subsection{Macro Geometry of Air: A Distributed Topology}

Whereas the key objective of the research aims towards a recalibration of geometry and material functionalities of air-distribution systems towards improved environmental impact, it also further aligns with the provision of human thermal comfort as "expressed satisfaction", noted as a principal purpose of HVAC systems (ASHRAE 2005).

Furthermore, a direct correlation exists between the: airflow in an airdistribution system, spatial set-out, differentiated occupation zones, and the resulting thermal comfort of the occupant. An initial performance study of HVAC ducts was undertaken through a Computational Fluid Dynamic (CFD) simulation, in order to provide an overview of spatial and temporal behavioural characteristics of air. Applied to a standard room volume (FFL to soffit of $3150 \mathrm{~mm}$ ), the cross-sectional study in Figure 4.a illustrates the impacts of location and distances of vents and diffusers in an open plan environment, demonstrating differentiations for localised air velocities, distribution, diffusion and temperature gradients. Detailed CFD studies into air velocity through a duct and bifurcation node suggested that dimensions for cross sections after bifurcation could be substantially reduced (Figure 4.b). A subsequent geometric prototype study provided the research with a matrix for bifurcation nodes that are optimised for airflow (Figure 4.c). 

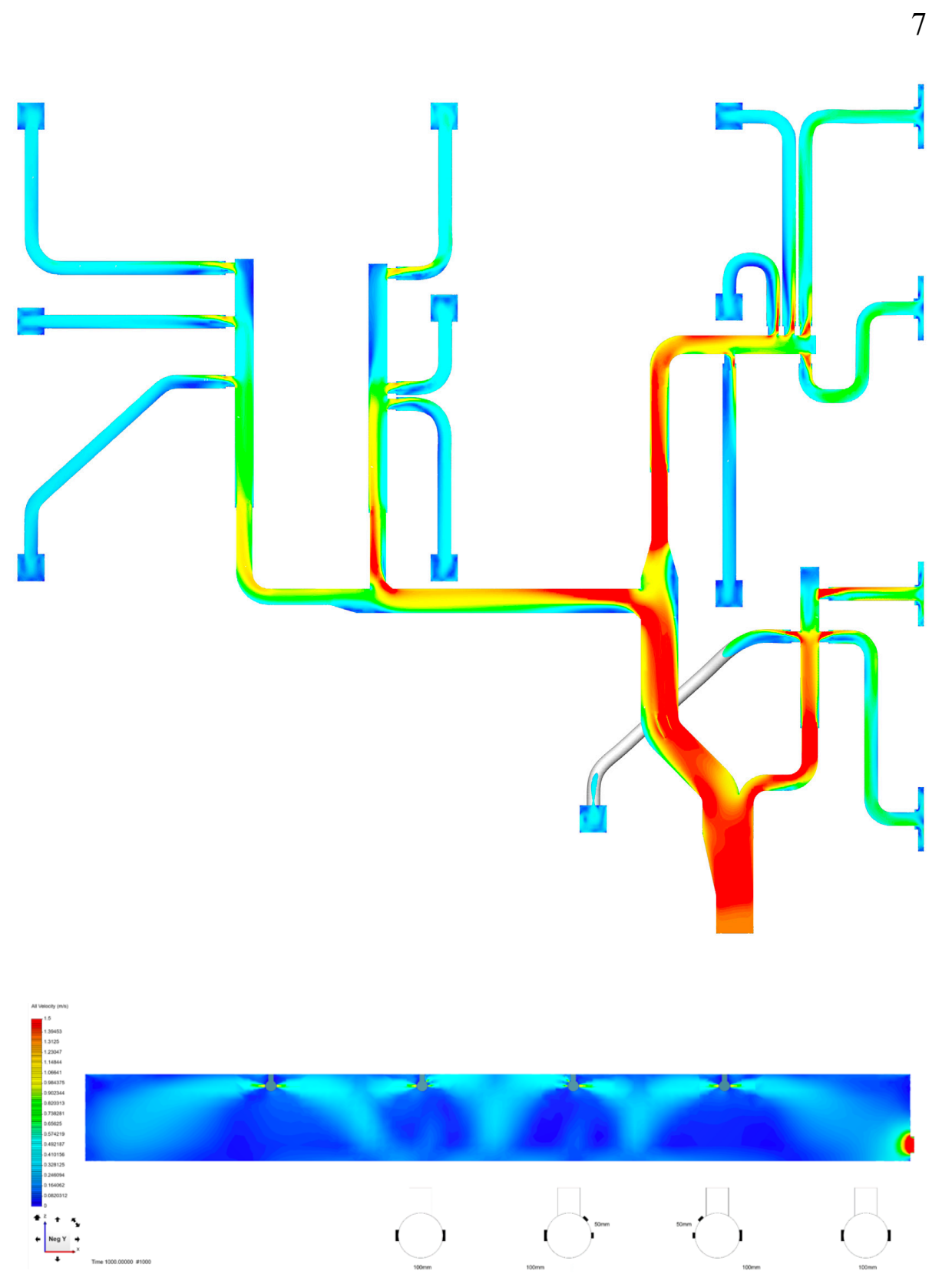


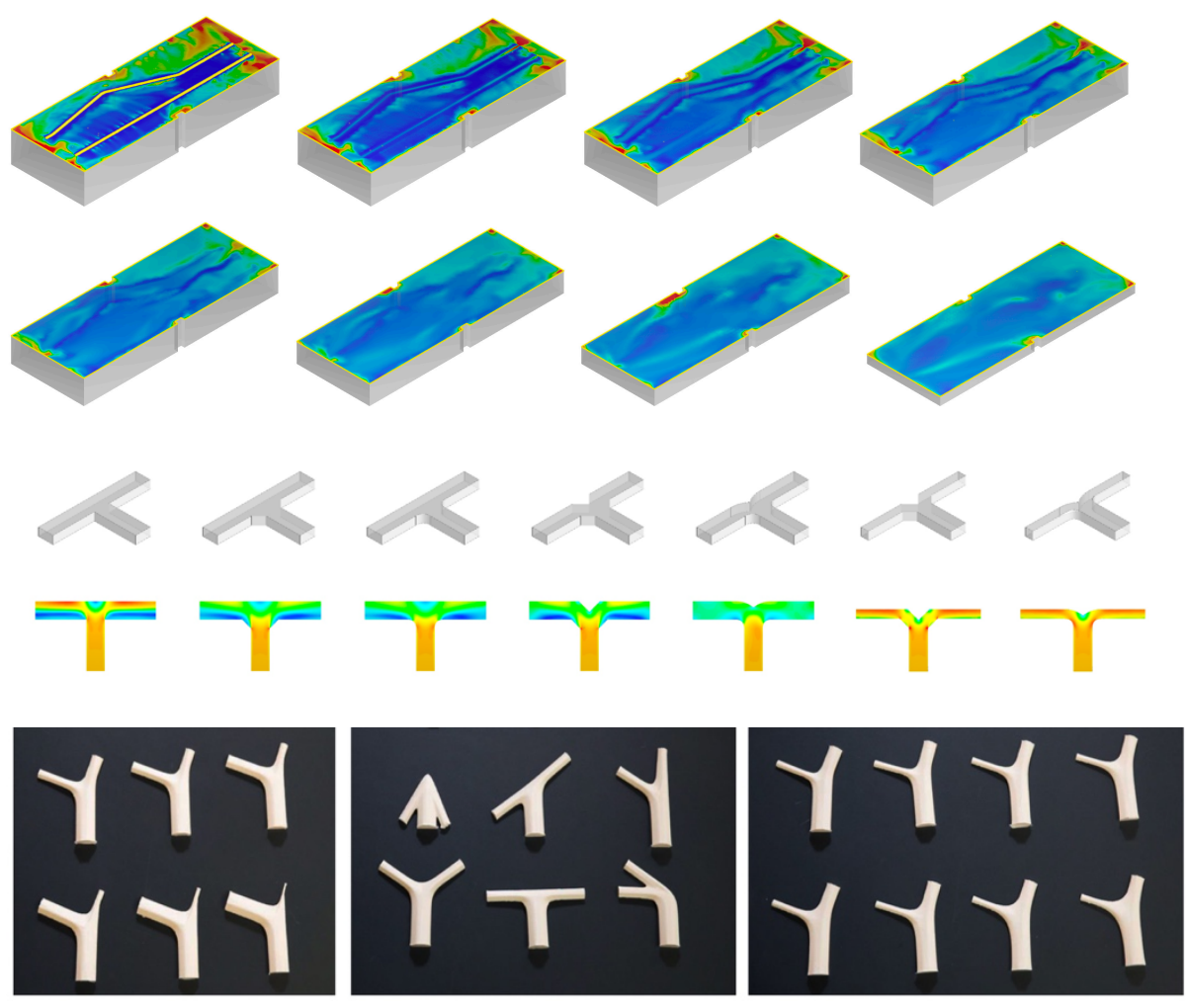

Fig. 3. Computational Fluid Dynamic (CFD) simulations in order to understand potential geometry organisation relative to behavioural characteristics of airflow (a) and air velocity study of bifurcation node (b) and 3D printed prototypes (c).

The resulting topological matrix for bifurcating systems was tested for a non-parallel robotic extrusion toolpath to investigate under which parameters bespoke cross sections that determine the global form could be achieved (see Figure 5). 

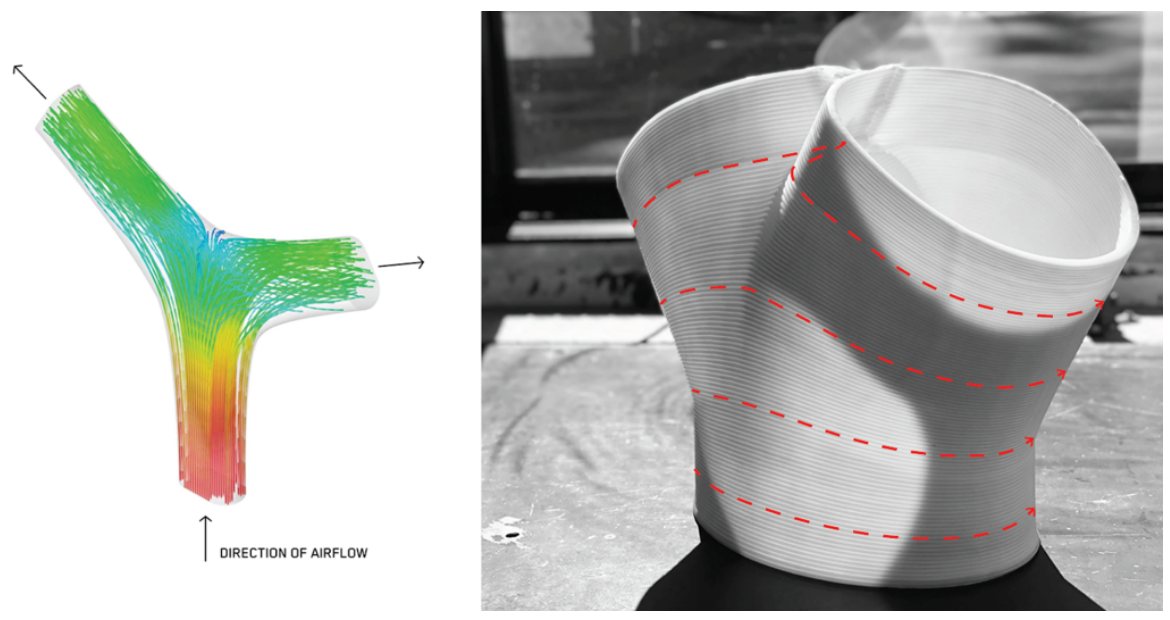

Fig. 4. CFD simulation of aerodynamic bifurcation (left) and corresponding 1:1 scale robotically printed prototype showing non-parallel extrusion toolpath to achieve desired global form (right).

The results of this initial study were encoded into a series of algorithmic procedures that were developed at a macro scale to take into account the existing on-site work and climatic zones of an open plan workplace, and to compute an ideal distributed topology (spatial layout) of the overall air-distribution system (see Figure 6).

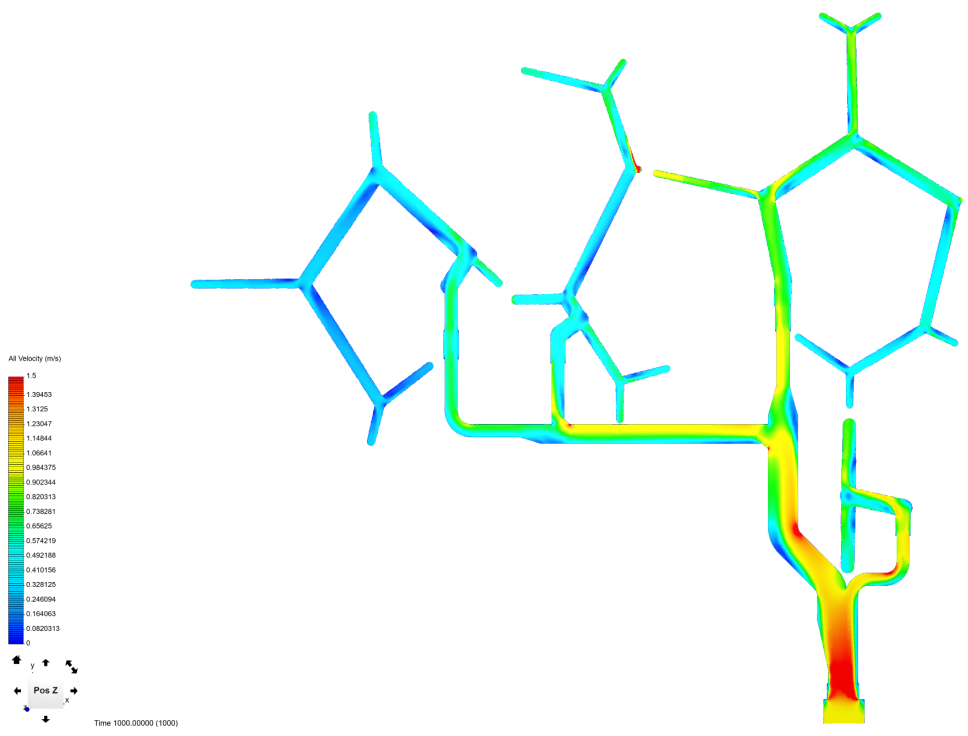



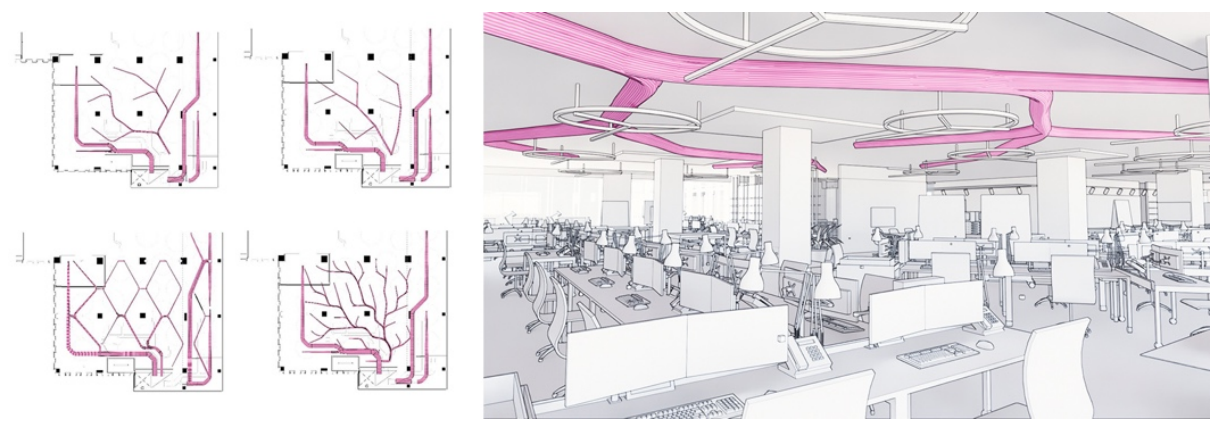

Fig. 5. Top: CFD simulation of initial investigation of branching topology in SimScale ${ }^{\mathrm{TM}}$ showing in-consistent air pressure across network. Bottom: Results of distribution algorithm (left) and visualisation of chosen solution in existing on-site context of open plan workplace (right).

\subsection{Meso Geometry of Air: Negotiated Morphologies}

On a meso level, this resulting topology is used as the input for a subsequent algorithmic procedure that accurately dimensions and sizes each branch's effective cross-section and required porosity at predefined intervals, in order to maintain the required air-supply and diffusion (Figure 7). The result is a continuously tapered and spatially differentiated bifurcating network with consistent air pressure that allows for a flexible and adaptable use of the office environment.

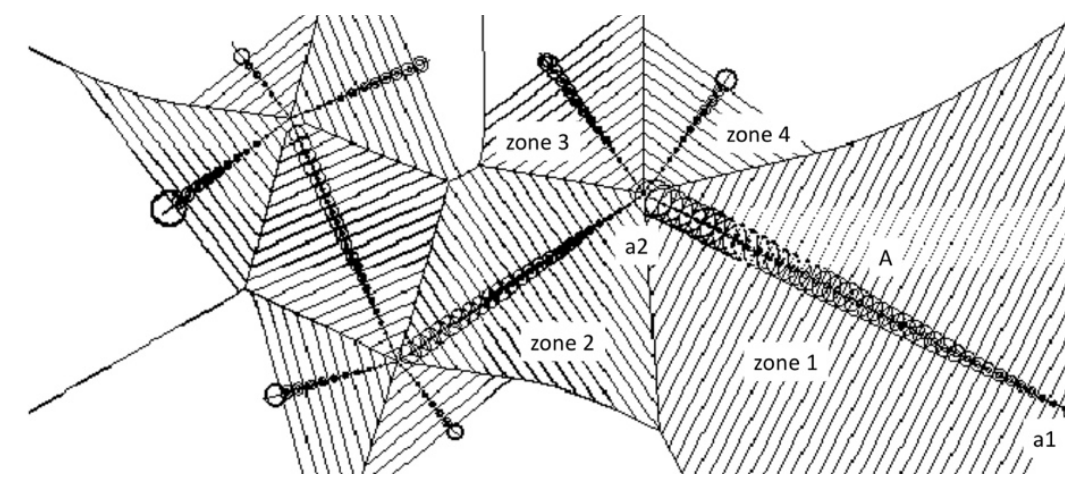

Fig. 6. Sizing of required tapering-effective cross section for duct profiles relative to desired climatic zones across floorplate (see duct cross section changes between a1 to a2). 


\subsection{The Micro Geometry of Air: Differentiated Porosity}

On a micro level, geometries of the 3D printed ductwork primarily integrate diffusion. This enables multiple performative qualities, providing aesthetic differentiation, textural variance, and crucially, the main diffusion strategy for the delivery of conditioned air. As opposed to traditional ducting systems, the diffuser is not a separate part, but instead is integrated in the overall system geometry. The flexibility of 3D printing allows the duct to be geometrically finely tuned, which in itself is a passive performative element as it allows the air distribution system to self-balance the pressure levels.

Significantly, the calibrated geometries allow for specified diffusion in respective zones. Multiple strategies to achieve effective diffusion range from low resolution textural variances, to high resolution porosity precisely deployed by the developed multioptimisation strategy. These micro diffusion geometries (see Fig. 8) are created by gestures encoded into the toolpath and robot trajectory. Jumps, velocity shifts, pauses, non-planar gestures, varied extrusion rates, and changes in extrusion cooling offer and achieve a broad range of controllable diffusion moments.

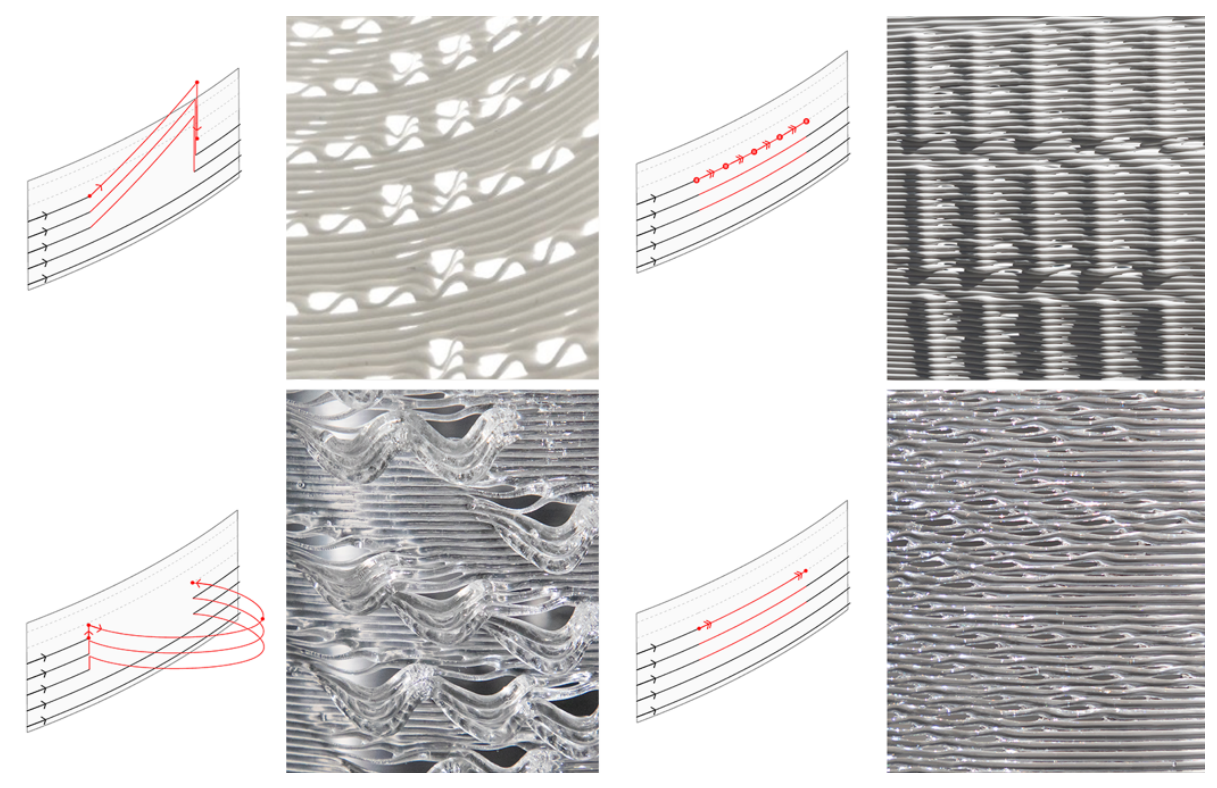

Fig. 7. Diffusion strategies as a result of robotic control and gestures shown in red. toolpath jumps [top left]; toolpath pauses [top right]; out of plane [bottom left]; micro-perforations as a result of speed and extrusion control [bottom right]. 


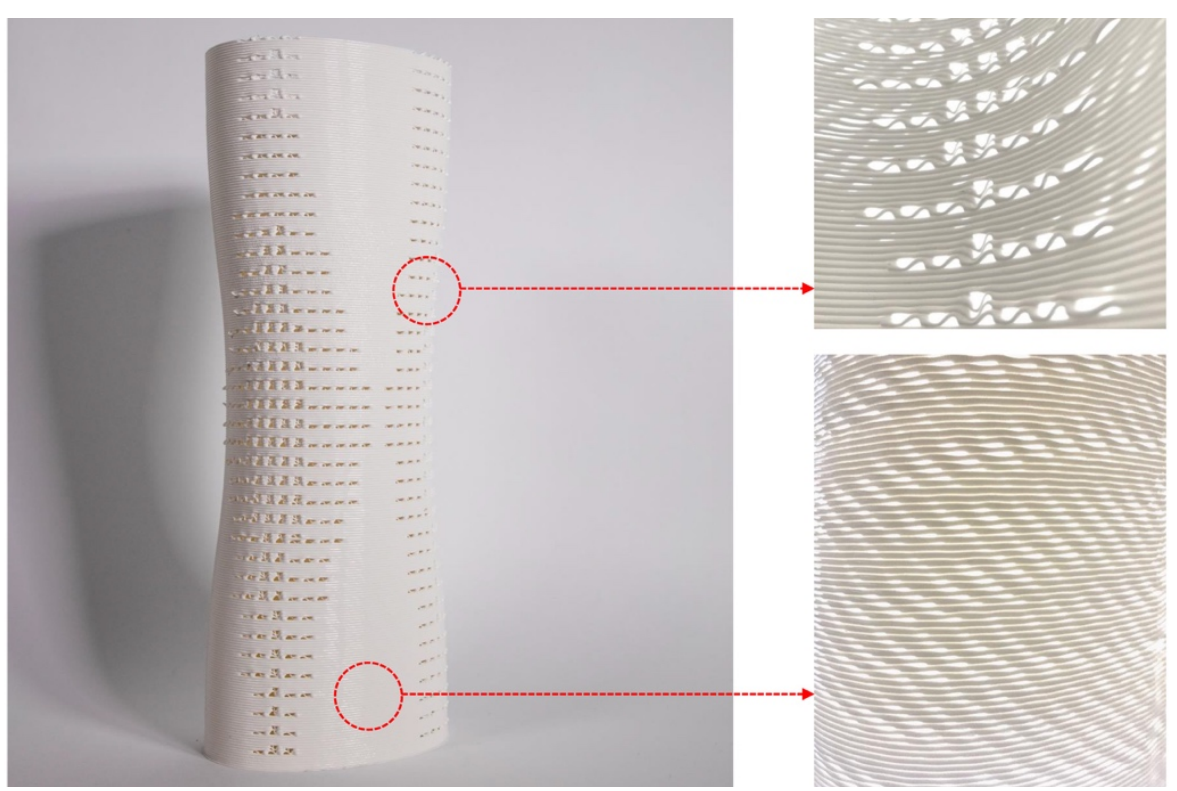

Fig. 8. Pilot: module element printed to onsite access limitations $(>2 \mathrm{~m})$, and accentuated diffusion zones as embedded surface characteristics.

A series of test geometries were printed to allow for an accurate evaluation and measuring of dimensional accuracy, layer compaction and shrinkage uniformity (see Fig. 9). The geometries also underwent physical tests with controlled velocities and airflow were conducted, demonstrating the diffusion of conditioned air via the implemented micro geometries. Fig. 10 visually demonstrates the continuously dynamic airstreams as a function of the pores integrated in the 3D robotic print.
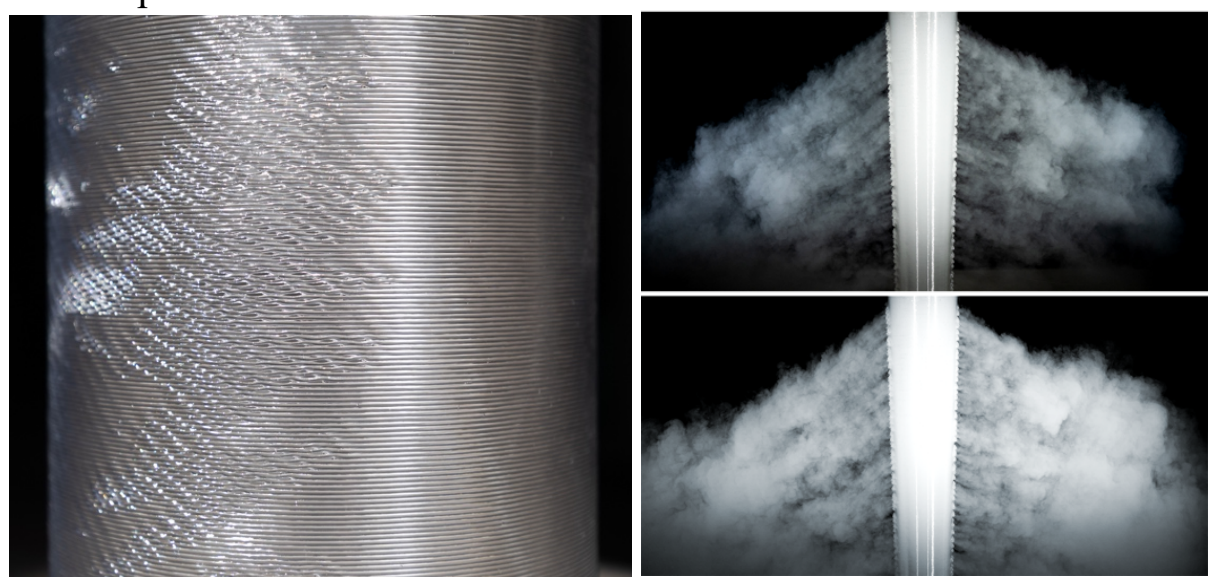
Fig. 9. The results of the air diffusion tests validate the approach

\subsection{Multi-objective optimisation}

The morphological and structural exploration of the air distribution network, where conflicting variables, including structural displacement and utilisation, material usage, wall thickness, cross-sectional attributes, and Gaussian curvature form the guiding framework and inputs for our developed multi-objective optimisation model, so that effective cross-section, structure, porosity and diffusion of the system can be co-evolved

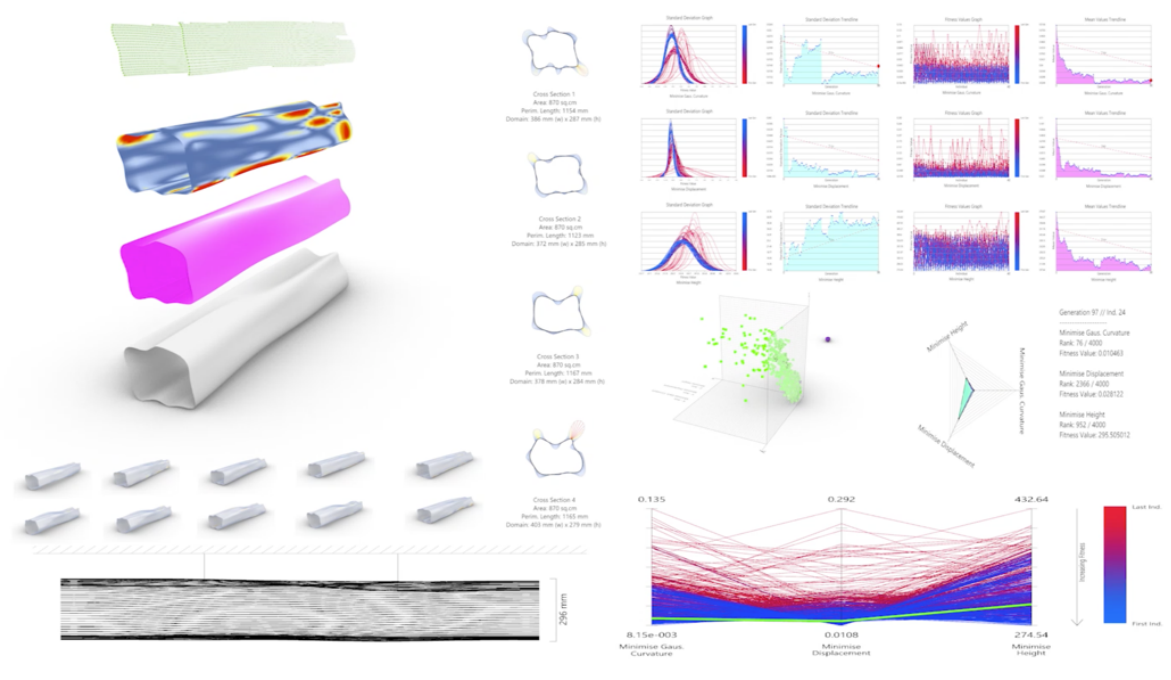

Fig. 10. Results of the multi-objective optimisation model, whereby project criteria include utilisation, material usage, thickness, cross-sectional attributes and gaussian curvature.

\subsection{Fabricating}

\subsection{Machine workflows and Fabrication setup}

In order to fully leverage the functional capacities of PETG we developed a fabrication system that consists of several integrated hardware and software sub-systems and enables a deep control of the material deposition at multiple levels of resolution. The generation of tool paths and robotic trajectory planning are achieved by custom written computational procedures and data workflows. In order to achieve the desired print quality a series of bespoke and material 
specific end effectors were developed and custom built by the interdisciplinary research team. The final design is able to achieve variable and reliable extrusion with PET-G (Polyethylene terephthalate, glycol modified) with a control system that allows a precise calibration of temperature, motor and gearbox. The system displayed in Figure 3 consists of two heating elements, positioned at the nozzle and adjustable along the barrel, which are controlled by two PID controllers that offer control of pre-melt and flow rates inside the extruder barrel. The custom built end effector also offers future experimentation with varied nozzle profiles to enhance or optimise extrusion rates and profiles. The tapered auger, flex coupler, and gearbox are driven by a Nema 42 stepper motor which is controllable either by a remote motor motion controller via pulse width modulation frequency, or via a programmable logic controller [PLC] system. Efficiency and reliability of print speed with PET-G also relies on rapid cooling to avoid excessive heat and print slump. This is achieved via a programmed activation of an air solenoid that directs compressed air to an array of cooling jets surrounding the 3D print tool path. Material is fed into the gravity fed hopper via a remote vacuum feed system that can be activated remotely to facility continuous printing.

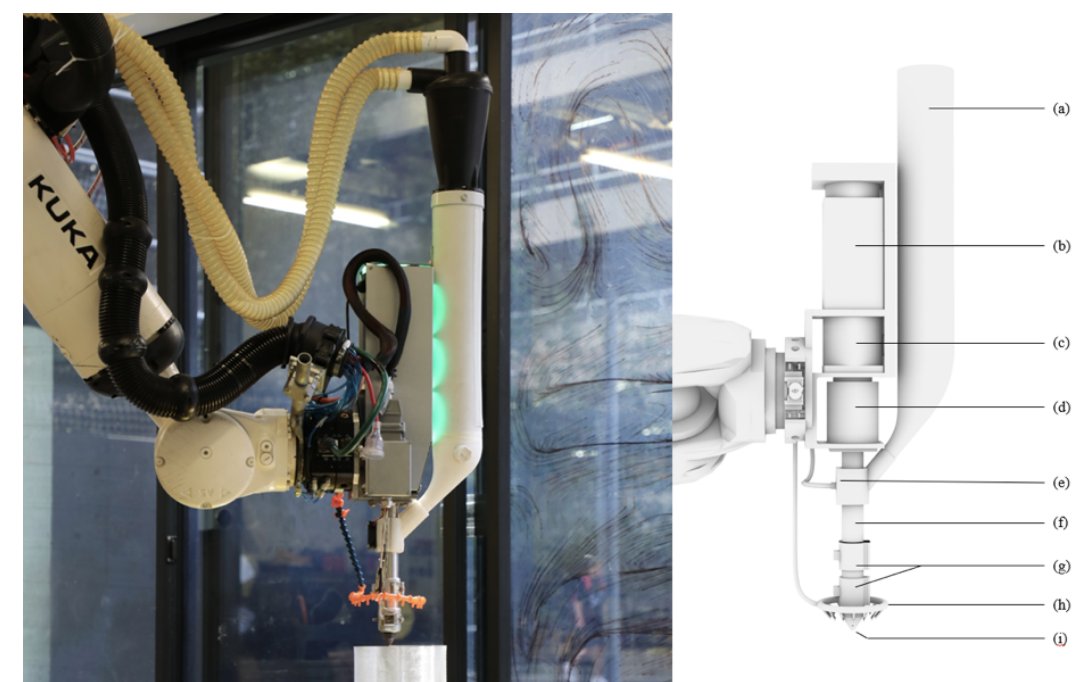

Fig. 11. Custom built end-effector and feed system showing a) gravity fed hopper, b) spindle, c) planetary gearbox, d) flex coupler, e) throat air cooling assembly, f) tapered auger and barrel assembly, g) thermocouplers, h) print cooling assembly i) $3 \mathrm{~mm}$ nozzle. 


\subsection{Customising material properties/character}

Working with PET-G affords the added benefit of mixing in additives in order to customise the material properties. Such additives can alter friction and slip coefficients, material hardness and clarity, static build up reduction, fire retardance and colour among others.
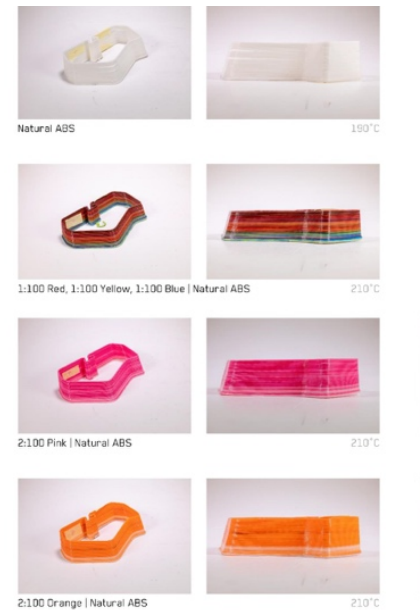
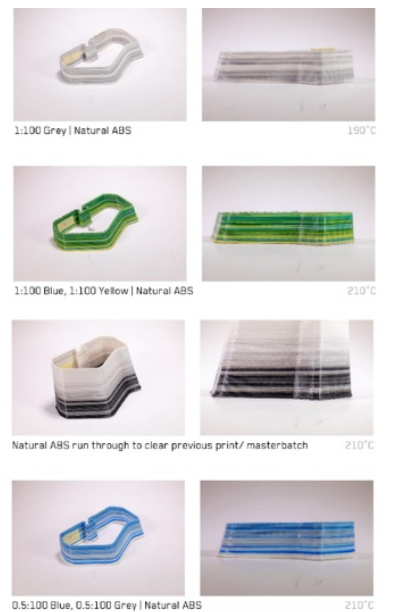

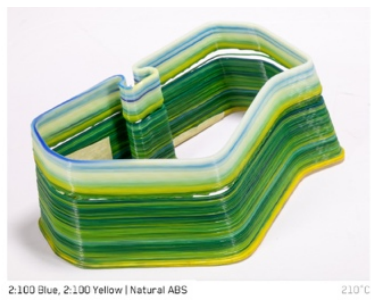

2.100 9lve, 2.100 Yellow| Katural Aes

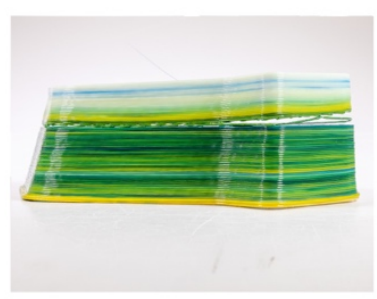

Fig. 12. Masterbatch colour study exploring varying mix ratios (left) and delamination due to masterbatch (right).

Within our study, we investigated colour as complementary aesthetic characteristics for ceilings in office environments. Hence, the project explored colour additives towards gradient expressions for duct modules (see Figures $13 \& 14$ ). This investigation into multi-coloured large-scale robotic $3 \mathrm{D}$ printing also extends current research in colour printing. 


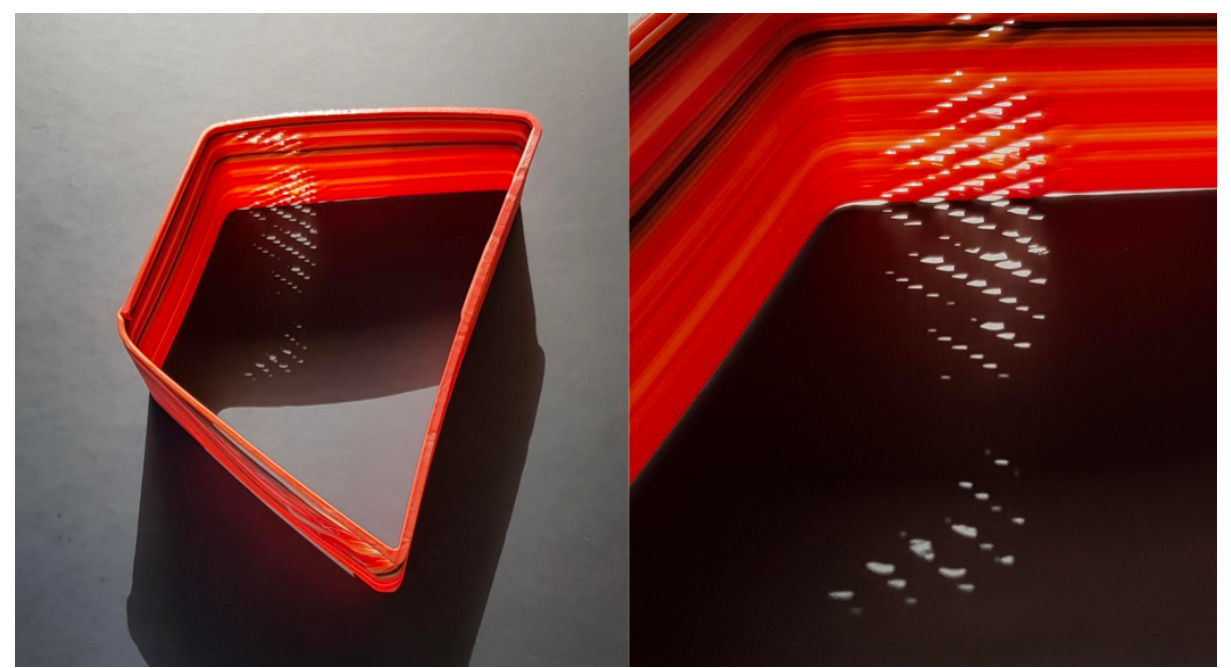

Fig. 13. Porosity study on trapezoidal and tapering cross-section with tailored colour gradient.

\subsection{Assembling the network}

Despite the build volume of the robotic workcell being significantly larger, we limited the maximum height of the 3D printed components to $2 \mathrm{~m}$ in order to ensure ease of transport to and on site. Connections between individual components are established through the use of a standard socket and spigot joint that is seamlessly integrated in the 3D printing process. This was achieved through the development of a bespoke printing method and the carefully tuned interplay between incrementally reducing the print layer height and robot velocity in conjunction with offsetting the robot trajectory. The combination of these results in thinner and wider print layers that create the required corbel for an air-tight connection between components (see Fig.12). 

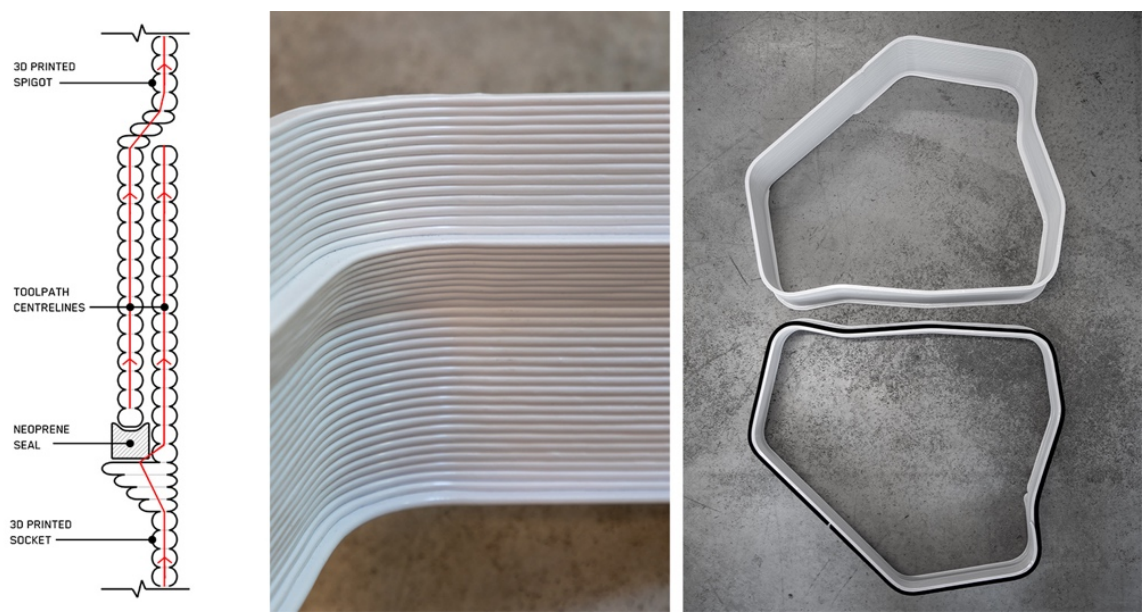

Fig. 14. Socket and spigot joint including neoprene seal showing different $3 \mathrm{D}$ printed layer heights and widths and robot trajectory in red.

\section{Conclusion and future work}

The research successfully demonstrates the way in which robotic 3D plastic extrusion can be adopted for the fabrication of highly tuned ductwork with integrated diffusion. This is demonstrated through testing the setup of micro geometries of continuous diffusion through tapered cross section. In addition to the CFD simulations of airflow and diffusion, a physical duct and diffusion test station was developed to ascertain proof of concept, and the real world, on-site performative aspects of the 3D printed ductwork in the pursuit of delivering enhanced and efficient thermal comfort. The outcome of this project illustrates how a novel approach to robotic large-scale 3D printing can act as a catalyst for innovative change in existing building practises, and drive environmental change in existing building systems. The scope of such positive environmental impacts, and the capacity to reduce the carbon emission footprint of the built environment, is enabled by the application of robotics by designers and architects. This project demonstrates how the disruption of existing practices, and the enabling of new material systems, is a critical step in the development of the built world in which we exist - and, most importantly, 
commences the conversation for an industry to begin to evolve to forward looking and sustainable practises.

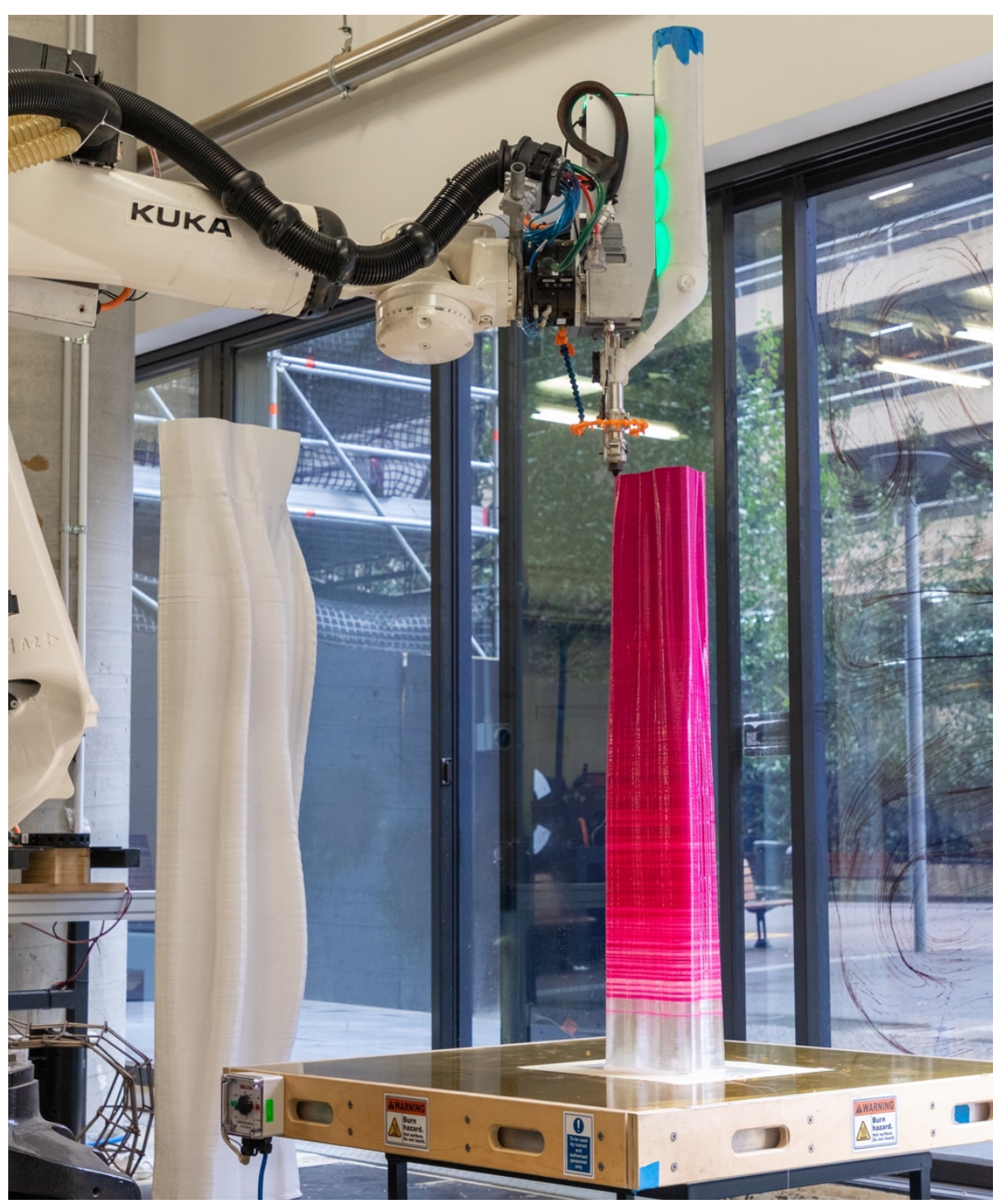

Fig. 15. Fabrication of component for pilot project.

On a technological level, this research extends existing knowledge by showcasing how a strategic integration and manipulation of multiple variables, such as toolpaths, robot trajectory, extrusion rate, temperature, and speed, enables the fabrication of topologically 
complex and highly intricate geometries, without the need of additionally printed support structure. Additionally, this research highlights the broad range of interdisciplinary conceptual processes, which are dissected and integrated into an architecturally deployed robotic approach to fabrication.

On a material system level, the shift from a thermally conductive metal, to a thermally insulating plastic - which has exponential growth for recycled streams - has additional environmental benefits as it eliminates the need for thermal lagging, and therefore further reduces the material and embodied carbon footprint of the system.

Across the different robotic test series, a number of results could be achieved, as is discussed in following:

- To test the validity of the approach, quality experiments have been conducted with a bespoke testing facility.

- To prove the printed prototypes reached the required diffusion, physical smoke tests were conducted.

- Several experiments have been conducted to collect data during the printing process.

- Various approaches to the addition of variegated colour have been tested.

- Varying pore geometries were tested in a physical testing facility at varying air velocities.

- The quality and thickness of 3D extrusion could be successfully controlled to produce lightweight, robust ducts with variable custom cross sections, air-tight joints, integrated features for suspension and aerodynamic bifurcation of module sections.

A number of limitations should be noted though:

- Due to the nature of the material and the necessity of a controlled environment, the employed fabrication process is more suitable for the prefabrication of building components in a factory, rather than direct on-site construction.

- A combination of more accurate sizing, material and geometry changes to the system could directly reduce the embodied energy/carbon. 
This paper has discussed empirical research into new differentiated material robotic plastic extrusion of modules for an air-distribution system. By integrating computational fluid dynamic simulation and multi-objective optimisation processes, highly-detailed geometries across macro, meso and micro scales could be derived that are optimised for air distribution in support of human-centred, thermally comfortable and locally differentiated zones within flexible open office spaces.

\section{References}

3DFuel. 2020. https://www.3dfuel.com/products/rpetg.

ASHRAE. 2005. Handbook: Fundamentals - SI edition. Atlanta, Ga.:

American Society of Heating Refrigerating and AirConditionin.

Bhate, Dhruv. 2015. "3D Printed Plastics in Functional Aerospace Parts." accessed 17 July.

Helm, Volker, Jan Willmann, Andreas Thoma, Luka Piskorec, Norman Hack, Fabio Gramazio, and Matthias Kohler. 2015. "Iridescence Print: Robotically Printed Lightweight Mesh Structures." 3D Printing and Additive Manufacturing 2:117-122. doi: 10.1089/3dp.2015.0018.

Kim, Taeyoung, and Yong-Gu Lee. 2018. "Shape transformable bifurcated stents." Scientific reports 8 (1):13911-13911. doi: 10.1038/s41598-018-32129-3.

Kuzmenko, Kateryna, Nadja Gaudilliere, Adélaïde Feraille, Justin Dirrenberger, and Olivier Baverel. 2019. "Assessing the Environmental Viability of 3D Concrete Printing Technology." Design Modelling Symposium - Impact: Design with All Senses.

Lyons, Brett P. 2014. "Additive Manufacturing in Aerospace: Examples and Research Outlook." Frontiers of Engineering 42 (1):13-19.

Nagami. https://www.nagami.design/en/.

Noller, Caroline. 2020. The Green Book. Sydney: The Footprint Company.

Pataky, Kris, Thomas Braschler, Andrea Negro, Philippe Renaud, Matthias Lutolf, and Juergen Brugger. 2012. "Microdrop 
Printing of Hydrogel Bioinks into 3D Tissue-Like Geometries." Advanced materials (Deerfield Beach, Fla.) 24. doi: 10.1002/adma.201102800.

Singamneni, Sarat, Yifan Lv, Andrew Hewitt, Rodger Chalk, Wayne Thomas, and David Jordison. 2019. "Additive Manufacturing for the Aircraft Industry: A Review." 8:1. doi: 10.4172/23296542.1000214.

Snooks, Studio. 2019. http://www.rolandsnooks.com/\#/sensilab-studio/. 